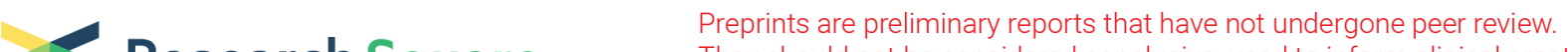

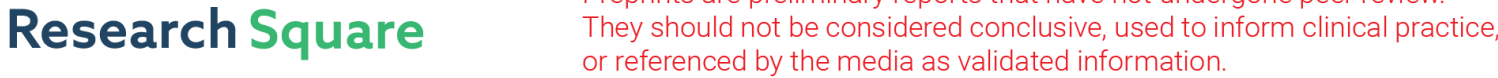 \\ Determinants of SARS-CoV-2 Infection in the Older Adult Population: Data From the LOST in Lombardia Study
}

\section{Giansanto Mosconi}

Department of Public Health, Experimental and Forensic Medicine, University of Pavia, Pavia

\section{Chiara Stival}

Department of Environmental Health Sciences, Istituto di Ricerche Farmacologiche Mario Negri IRCCS, Milan

\section{Alessandra Lugo}

Department of Environmental Health Sciences, Istituto di Ricerche Farmacologiche Mario Negri IRCCS, Milan

\section{Carlo Signorelli}

School of Medicine, University Vita-Salute San Raffaele, Milan

\section{Andrea Amerio}

Department of Neuroscience, Rehabilitation, Ophthalmology, Genetics, Maternal and Child Health (DINOGMI), Section of Psychiatry, University of Genoa, Genoa

\section{Luca Cavalieri}

ATS della Brianza

\section{Licia lacoviello}

Research Center in Epidemiology and Preventive Medicine (EPIMED), University of Insubria

\section{David Stuckler}

Department of Social Sciences and Politics, Bocconi University, Milan

\section{Alberto Zucchi}

ATS di Bergamo

\section{Anna Odone ( $\nabla$ anna.odone@unipv.it )}

Department of Public Health, Experimental and Forensic Medicine, University of Pavia, Pavia

\section{Silvano Gallus}

Department of Environmental Health Sciences, Istituto di Ricerche Farmacologiche Mario Negri IRCCS, Milan

\section{Research Article}

Keywords: 
Posted Date: February 11th, 2022

DOI: https://doi.org/10.21203/rs.3.rs-1327147/v1

License: (c) (i) This work is licensed under a Creative Commons Attribution 4.0 International License. Read Full License 


\section{Abstract}

Most COVID-19-related fatalities occurred among older adults, however to date, evidence on determinants of SARS-CoV-2 infection in this population is limited and mostly based on case series without a comparison group. A telephone-based cross-sectional study was conducted in November 2020 on a representative sample of 4,400 Italians aged $\geq 65$ years residing in the Lombardy region. We determined the prevalence of a history of SARS-CoV-2 infection in the period between the beginning of the pandemic and the time of the interview. Thorough unconditional multiple logistic regression models, we estimated odds ratios (OR) of SARS-CoV-2 infection and the corresponding $95 \%$ confidence intervals $(\mathrm{Cl})$. We also evaluated if the infection was a determinant of a worsening in mental health wellbeing. Overall, $4.9 \%$ of participants reported a history of SARS-CoV-2 infection. No significant relationship between sex and SARS-CoV-2 infection was observed. SARS-CoV-2 infection was less frequently reported in subjects aged $\geq 70$ (OR=0.55; 95\% 0.41-0.74) compared to 65-69 years. No trend was observed after 70 years of age. Those with at least one chronic condition reported a lower infection rate compared to healthy subjects (OR=0.68 95\% Cl: 0.49-0.93). Separated/divorced participants less frequently reported infection than married/cohabiting ones (OR=2.33 95\% Cl: 1.29-4.20). Self-reported history of SARS-CoV-2 infection resulted being a determinant of an increase in depressive symptoms (OR=1.57; 95\% Cl: 1.17-2.10). This first large representative study of people aged 65 years or over suggests that in Italy the oldest subjects and those having chronic conditions less frequently expose themselves to SARS-CoV-2 infection during the pandemic.

\section{Introduction}

During the first wave of COVID-19 contagion, Italy's Lombardy region, among the first hit in western countries, experienced high levels of hospitals congestion and a relevant COVID-19 case fatality rate $\mathrm{e}^{1-3}$. By the end of 2020, this region had registered the highest number of infections in the country, as well as one of the highest cumulative incidence rates of infection ${ }^{1,4}$

According to the national-level COVID-19 surveillance system, the cumulative incidence of SARS-CoV-2 infection in Italy in 2020 (before vaccines were available) showed an increasing trend with increasing age, and by the end of the same year, $96 \%$ and $86 \%$ of COVID-19-related fatalities had occurred among people aged 60 and 70 years and over, respectively ${ }^{4}$. It is well documented in the literature how advanced age, being male and the presence of chronic diseases are risk factors for adverse outcomes in COVID-19 patients ${ }^{5,6}$.

To date, knowledge about potential determinants of SARS-CoV-2 infection is mostly based on case series (inappropriate to investigate the issue not having a comparison group), or surveys enrolling convenience samples (providing findings not extensible to the general population) ${ }^{7}$. The few representative crosssectional studies were online surveys which are unsuitable for examining those populations, like the elderly, that often do not have internet access or have a low level of computer skills ${ }^{8}$. 
To the best of our knowledge, this is the first study that evaluates the prevalence of individuals selfreporting a history of SARS-CoV-2 infection and investigates its possible determinants in a representative sample of Italian home-dwelling people aged 65 years or older.

\section{Results}

In November 2020, out of 4,400 participants, 213 (4.9\%) reported to have been infected by SARS-CoV-2. In particular, 98 participants $(2.2 \%)$ received a diagnosis through a positive rhino pharyngeal swab test, 46 $(1.1 \%)$ through positive serological test and $70(1.6 \%)$ had experienced signs or symptoms without a confirmed diagnosis (Figure 1).

Table 1 shows the ORs of subjects reporting SARS-CoV-2 infection by selected characteristics. No relationship was observed between sex and infection (compared to women, OR for men was $1.02 ; 95 \% \mathrm{Cl}$ : 0.77-1.36). Subjects aged 70 years and older reported SARS-CoV-2 infection less frequently than those aged $65-69$ years (OR=0.55; $95 \% \mathrm{Cl}: 0.41-0.74)$. When considering participants aged 70 years or more no trend of infection was observed with increasing age ( $p$ for trend $=0.447)$. Compared to participants from Milan (Lombardy region's capital city), participants from Bergamo and Brescia more frequently reported SARS-CoV-2 infection (OR=2.11; 95\% Cl: 1.46-3.06). Divorced and separated subjects reported to have been infected more frequently compared to married ones or those living with a partner $(\mathrm{OR}=2.33 ; 95 \% \mathrm{Cl}$ : 1.29-4.20). 
Table 1

Distribution of 4,400 Italian subjects aged 65 years or more according to SARS-CoV- 2 infection, overall and by selected characteristics. Corresponding odds ratios ${ }^{\wedge}(\mathrm{OR})$ and $95 \%$ confidence intervals $(\mathrm{Cl})$. Lombardy region, Italy 2020.

\begin{tabular}{|c|c|c|c|c|c|}
\hline \multirow[t]{2}{*}{ Characteristics } & \multirow[t]{2}{*}{$\mathbf{N}$} & \multicolumn{2}{|c|}{$\begin{array}{l}\text { SARS-CoV-2 infection based on } \\
\text { confirmed diagnosis or symptoms }\end{array}$} & \multicolumn{2}{|c|}{$\begin{array}{l}\text { SARS-CoV-2 infection based } \\
\text { on confirmed diagnosis only* }\end{array}$} \\
\hline & & $\%$ & OR $(95 \% \mathrm{Cl})$ & $\%$ & OR $(95 \% \mathrm{Cl})$ \\
\hline Total & 4400 & 4.9 & & 3.3 & \\
\hline \multicolumn{6}{|l|}{ Sex } \\
\hline Women & 2498 & 4.7 & $1.00^{\circ}$ & 3.1 & $1.00^{\circ}$ \\
\hline Men & 1902 & 5.0 & $1.02(0.77-1.36)$ & 3.6 & $1.11(0.79-1.56)$ \\
\hline \multicolumn{6}{|l|}{ Age group (years) } \\
\hline $65-69$ & 1289 & 7.3 & $1.00^{\circ}$ & 5.6 & $1.00^{\circ}$ \\
\hline $70-74$ & 838 & 3.2 & $0.45(0.29-0.70)$ & 2.3 & $0.41(0.24-0.70)$ \\
\hline $75-79$ & 1188 & 4.3 & $0.62(0.43-0.88)$ & 2.8 & $0.52(0.33-0.80)$ \\
\hline $80+$ & 1085 & 3.8 & $0.57(0.38-0.86)$ & 2.1 & $0.41(0.25-0.69)$ \\
\hline$P$ for trend & & & 0.006 & & $<0.001$ \\
\hline \multicolumn{6}{|l|}{ Level of education } \\
\hline Low & 788 & 3.7 & $1.00^{\circ}$ & 2.5 & $1.00^{\circ}$ \\
\hline Intermediate & 3168 & 5.2 & $1.20(0.78-1.84)$ & 3.5 & $1.04(0.62-1.74)$ \\
\hline High & 444 & 4.3 & $0.92(0.50-1.71)$ & 3.1 & $0.81(0.39-1.67)$ \\
\hline$P$ for trend & & & 0.914 & & 0.592 \\
\hline \multicolumn{6}{|l|}{ Province } \\
\hline Ml & 1424 & 3.5 & $1.00^{\circ}$ & 2.4 & $1.00^{\circ}$ \\
\hline $\mathrm{CO}+\mathrm{LC}+\mathrm{MB}+\mathrm{VA}+\mathrm{SO}$ & 1262 & 4.8 & $1.34(0.91-1.97)$ & 3.2 & $1.24(0.77-1.98)$ \\
\hline$B G+B S$ & 1038 & 7.2 & $2.11(1.46-3.06)$ & 4.6 & $1.87(1.19-2.95)$ \\
\hline$C R+L O+M N+P V$ & 677 & 4.1 & $1.11(0.69-1.79)$ & 3.5 & $1.35(0.79-2.31)$ \\
\hline
\end{tabular}

^ Estimated by unconditional multiple logistic regression models after adjustment for sex, age, education level, province and number of chronic conditions; estimates in bold are those statistically significant at 0.05 level.

* 70 subjects reporting COVID-19 symptoms but no diagnosis were excluded from the analyses.

- Reference category 


\begin{tabular}{|c|c|c|c|c|c|}
\hline \multirow[t]{2}{*}{ Characteristics } & \multirow[t]{2}{*}{$\mathbf{N}$} & \multicolumn{2}{|c|}{$\begin{array}{l}\text { SARS-CoV-2 infection based on } \\
\text { confirmed diagnosis or symptoms }\end{array}$} & \multicolumn{2}{|c|}{$\begin{array}{l}\text { SARS-CoV-2 infection based } \\
\text { on confirmed diagnosis only* }\end{array}$} \\
\hline & & $\%$ & OR $(95 \% \mathrm{Cl})$ & $\%$ & OR $(95 \% \mathrm{Cl})$ \\
\hline \multicolumn{6}{|l|}{ Marital status } \\
\hline $\begin{array}{l}\text { Married \Living with } \\
\text { a partner }\end{array}$ & 3113 & 4.7 & $1.00^{\circ}$ & 3.3 & $1.00^{\circ}$ \\
\hline Divorced/separated & 140 & 10.0 & $2.33(1.29-4.20)$ & 5.4 & $1.66(0.75-3.67)$ \\
\hline Widowed & 871 & 4.3 & $1.16(0.77-1.73)$ & 2.7 & $1.19(0.73-1.95)$ \\
\hline Never married & 276 & 5.5 & $1.06(0.61-1.84)$ & 4.1 & $1.10(0.58-2.08)$ \\
\hline \multicolumn{6}{|c|}{$\begin{array}{l}\text { ^ Estimated by unconditional multiple logistic regression models after adjustment for sex, age, } \\
\text { education level, province and number of chronic conditions; estimates in bold are those statistically } \\
\text { significant at } 0.05 \text { level. }\end{array}$} \\
\hline \multicolumn{6}{|c|}{ * 70 subjects reporting COVID-19 symptoms but no diagnosis were excluded from the analyses. } \\
\hline${ }^{\circ}$ Reference category & & & & & \\
\hline
\end{tabular}

Compared to those with no diseases, subjects with one or more chronic conditions less frequently reported SARS-CoV-2 infection (OR= 0.68; 95\% Cl: 0.49-0.93). No trend was observed with increasing number of chronic diseases ( $p$ for trend=0.112). People with diabetes less frequently reported SARS-CoV2 infection, compared to subjects without that disease (OR=0.64; $95 \% \mathrm{Cl}: 0.42-0.99)$, whereas people with migraine more frequently reported SARS-CoV-2 infection, compared to those without that condition (OR=2.01; 95\% Cl: 1.21-3.34; Table 2). 
Table 2

Distribution of 4,400 Italian subjects aged 65 years or more according to SARS-CoV-2 infection, overall and by chronic conditions. Corresponding odds ratios ${ }^{\wedge}(\mathrm{OR})$ and $95 \%$ confidence intervals (CI). Lombardy region, Italy 2020.

\begin{tabular}{|c|c|c|c|c|c|}
\hline \multirow[t]{2}{*}{ Characteristics } & \multirow[t]{2}{*}{$\mathbf{N}$} & \multicolumn{2}{|c|}{$\begin{array}{l}\text { SARS-CoV-2 infection with confirmed } \\
\text { diagnosis or symptoms }\end{array}$} & \multicolumn{2}{|c|}{$\begin{array}{l}\text { SARS-CoV-2 infection with } \\
\text { confirmed diagnosis only* }\end{array}$} \\
\hline & & $\%$ & OR (95\% Cl) & $\%$ & OR (95\% Cl) \\
\hline Total & 4400 & 4.9 & & 3.3 & \\
\hline \multicolumn{6}{|l|}{$\begin{array}{l}\text { Chronic } \\
\text { diseases }^{\dagger}\end{array}$} \\
\hline No & 890 & 6.9 & 1.00 & 5.1 & $1.00^{\circ}$ \\
\hline Yes & 3510 & 4.3 & $0.68(0.49-0.93)$ & 2.9 & $0.63(0.43-0.92)$ \\
\hline 1 & 1264 & 4.6 & $0.70(0.48-1.01)$ & 3.4 & $0.70(0.45-1.08)$ \\
\hline 2 & 1375 & 3.8 & $0.61(0.41-0.90)$ & 2.4 & $0.53(0.33-0.85)$ \\
\hline $3+$ & 870 & 4.6 & $0.76(0.50-1.17)$ & 2.9 & $0.68(0.40-1.14)$ \\
\hline$P$ for trend & & & 0.112 & & 0.043 \\
\hline Diabetes $^{\dagger}$ & 936 & 3.2 & $0.64(0.42-0.99)$ & 2.2 & $0.71(0.42-1.21)$ \\
\hline Hypertension $^{\dagger}$ & 2443 & 4.2 & $0.92(0.63-1.35)$ & 2.8 & $1.00(0.63-1.59)$ \\
\hline $\begin{array}{l}\text { Osteoarthritis } \\
\text { or arthritis }{ }^{\dagger}\end{array}$ & 1485 & 4.6 & $1.18(0.82-1.71)$ & 3.1 & $1.39(0.88-2.19)$ \\
\hline Asthma $^{\dagger}$ & 148 & 6.2 & $1.47(0.73-2.97)$ & 5.0 & $1.80(0.81-3.98)$ \\
\hline Cancer $^{\dagger}$ & 170 & 5.8 & $1.50(0.76-2.96)$ & 1.9 & $0.70(0.22-2.25)$ \\
\hline Heart disease $^{\dagger}$ & 539 & 4.3 & $1.07(0.66-1.72)$ & 2.6 & $1.05(0.57-1.91)$ \\
\hline Osteoporosis ${ }^{\dagger}$ & 652 & 4.6 & $1.10(0.70-1.74)$ & 2.8 & $1.05(0.59-1.87)$ \\
\hline Kidney failure $^{\dagger}$ & 95 & 6.0 & $1.53(0.63-3.75)$ & 2.2 & $0.86(0.20-3.65)$ \\
\hline Bronchitis $^{\dagger}$ & 188 & 4.4 & $0.96(0.46-2.01)$ & 2.9 & $0.96(0.39-2.39)$ \\
\hline \multicolumn{6}{|c|}{$\begin{array}{l}\text { ^ Estimated by unconditional multiple logistic regression models after adjustment for sex, age, } \\
\text { education level, province and number of chronic conditions; estimates in bold are those statisticall } \\
\text { significant at } 0.05 \text { level. }\end{array}$} \\
\hline \multicolumn{6}{|c|}{ * 70 subjects reporting COVID-19 symptoms but no diagnosis were excluded from the analyses. } \\
\hline \multicolumn{6}{|c|}{ • Reference category } \\
\hline † Diagn & 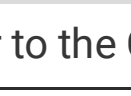 & 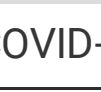 & emic breakout. & & \\
\hline
\end{tabular}




\begin{tabular}{|c|c|c|c|c|c|}
\hline \multirow[t]{2}{*}{ Characteristics } & \multirow[t]{2}{*}{$\mathbf{N}$} & \multicolumn{2}{|c|}{$\begin{array}{l}\text { SARS-CoV-2 infection with confirmed } \\
\text { diagnosis or symptoms }\end{array}$} & \multicolumn{2}{|c|}{$\begin{array}{l}\text { SARS-CoV-2 infection with } \\
\text { confirmed diagnosis only* }\end{array}$} \\
\hline & & $\%$ & OR $(95 \% \mathrm{Cl})$ & $\%$ & OR $(95 \% \mathrm{Cl})$ \\
\hline Migraine $^{\dagger}$ & 252 & 8.5 & $2.01(1.21-3.34)$ & 4.6 & $1.52(0.78-2.98)$ \\
\hline \multicolumn{6}{|c|}{$\begin{array}{l}\text { ^ Estimated by unconditional multiple logistic regression models after adjustment for sex, age, } \\
\text { education level, province and number of chronic conditions; estimates in bold are those statistically } \\
\text { significant at } 0.05 \text { level. }\end{array}$} \\
\hline \multicolumn{6}{|c|}{ * 70 subjects reporting COVID-19 symptoms but no diagnosis were excluded from the analyses. } \\
\hline \multicolumn{6}{|c|}{${ }^{\circ}$ Reference category } \\
\hline † Diagnosed pric & the & VID- & demic breakout. & & \\
\hline
\end{tabular}

Table 3 shows the OR of a worsening in anxiety and depressive symptoms during COVID-19 pandemic, according to SARS-CoV-2 infection. No relationship was observed between infection and an increase in anxiety symptoms, whereas an increase in depressive symptoms was more frequently reported in individuals who contracted the SARS-CoV-2 infection (OR=1.57; 95\% Cl: 1.17-2.10). The sensitivity analysis produced comparable results.

Table 3

Distribution of 4,400 Italian subjects aged 65 years or more according to a worsening in anxiety and depressive symptoms, by to SARS-CoV-2 infection. Corresponding odds ratios ${ }^{\wedge}(\mathrm{OR})$ and $95 \%$ confidence intervals (Cl). Lombardy region, Italy 2020.

\begin{tabular}{|c|c|c|c|c|c|}
\hline \multirow[t]{2}{*}{ Characteristics } & \multirow[t]{2}{*}{$\mathbf{N}$} & \multicolumn{2}{|c|}{$\begin{array}{l}\text { Increased anxiety } \\
\text { symptoms during } \\
\text { COVID-19 pandemic }\end{array}$} & \multicolumn{2}{|c|}{$\begin{array}{l}\text { Increased depressive } \\
\text { symptoms during } \\
\text { CoVID-19 pandemic }\end{array}$} \\
\hline & & $\%$ & OR (95\% Cl) & $\%$ & OR (95\% Cl) \\
\hline $\begin{array}{l}\text { SARS-CoV-2 infection with confirmed } \\
\text { diagnosis or symptoms vs. no infection }\end{array}$ & 213 & 39.9 & $\begin{array}{l}1.13(0.85- \\
1.51)\end{array}$ & 37.8 & $\begin{array}{l}1.57(1.17- \\
2.10)\end{array}$ \\
\hline $\begin{array}{l}\text { SARS-CoV-2 infection with confirmed } \\
\text { diagnosis only* vs. no infection or } \\
\text { infection but no diagnosis }\end{array}$ & 144 & 44.3 & $\begin{array}{l}1.36(0.97- \\
1.90)\end{array}$ & 39.5 & $\begin{array}{l}1.68(1.19- \\
2.38)\end{array}$ \\
\hline \multicolumn{6}{|c|}{$\begin{array}{l}\text { ^ Estimated by unconditional multiple logistic regression models after adjustment for sex, age, } \\
\text { education level, province and number of chronic conditions; estimates in bold are those statistically } \\
\text { significant at } 0.05 \text { level. }\end{array}$} \\
\hline
\end{tabular}

\section{Discussion}

In November 2020, almost $5 \%$ of older people from Lombardy region reported having had signs or symptoms compatible with COVID-19 or having been tested positive for SARS-CoV-2 infection based on a nasopharyngeal swab or a serological test. No relationship between sex or level of education and SARS- 
CoV-2 infection was observed. Subjects aged 65-69 years more frequently reported to have been infected compared to older subjects. Having one or more chronic diseases were related to a lower rate of infection. SARS-CoV-2 infection resulted being a determinant of a worsening in depressive symptoms.

Based on our data, after almost one year from the beginning of the COVID-19 pandemic, $2.2 \%$ of adults aged 65 and older from the Lombardy region reported having had a positive nasopharyngeal swab. In November 2020, corresponding official data from the Italian National Health Institute showed that the cumulative incidence was around $3.5 \%$ in Italian adults aged 60 or more and around $3.8 \%$ in the Lombardy general population ${ }^{9}$. The lower prevalence of people who tested positive on a swab detected within our survey might be explained by the exclusion from our sample of the individuals residing in nursing homes, and subjects who died within the 2020 due to the infection.

However, it is interesting to note that the percentage of participants who have been infected rose by $50 \%$ (reaching 3.3\%) when adding those who declared having tested positive for the serological test, and potentially more than doubled (up to $4.9 \%$ ) when adding those who experienced signs or symptoms compatible with COVID-19 without any test confirmation. These results might be explained by the fact that, in Italy, in the early stages of the pandemic, the performance of nasopharyngeal swabs was almost exclusively addressed to patients in severe conditions, thus excluding a large number of individuals with none or mild signs or symptoms ${ }^{10}$. Accordingly, the Italian Ministry of Health conducted a seroprevalence study between May 25, 2020, and July 15, 2020, which revealed that 7.5\% (95\% Cl: $6.8-8.3)$ of the Lombardy general population had SARS-CoV-2 antibodies ${ }^{11}$, confirming the fact that the real cumulative incidence is underestimated when considering only nasopharyngeal swab data.

With regard to the determinants of infection, an important clarification must be made.

Since COVID-19 is characterized by non-specific signs and symptoms ${ }^{12}$ some of which can be very common in the elderly population ${ }^{13,14}$, as a sensitivity analysis, we excluded subjects reporting only signs or symptoms without a confirmed diagnosis.

We did not observe any significant relationship between sex and SARS-CoV-2 infection, although our data seem to suggest a marginally higher prevalence of infection in males. This is partly in line with a recent systematic review ${ }^{15}$, that found men having a slightly higher frequency of infection ${ }^{16,17}$.

We found a lower frequency of infection in participants aged 70 years or older than in participants aged 65-69 years. This result could be explained by the fact that the latter group included a higher proportion of working subjects, likely more exposed to SARS-CoV-2 infection because of more frequent social interactions. In addition, since social isolation increases with age ${ }^{18}$, it is conceivable that subjects aged 70 years or more, having had fewer interactions, were better able to protect themselves from possible opportunities for infection. It is also likely that older people, aware of the higher risks to which they were exposed, were more careful to comply with preventive measures ${ }^{19}$. Our finding is in line with a large systematic review and meta-analysis including 241 seroprevalence studies showing that the proportion 
of individuals aged 65 years or older with SARS-CoV-2 antibodies tended to decrease with age ${ }^{16}$. Also, data from an Italian Ministry of Health survey show a decrease in seroprevalence in the over-70 age group compared to the 60-69 age group ${ }^{11}$. Since our survey did not allow for the inclusion of those who died of COVID-19, we recalculated the prevalence in every age group considering the number of expected cases, which is obtained by adding the proportion of deaths per age group based on the case fatality rate provided by the National Health Institute referring to November $2020^{9}$. The cases distribution by age remained substantially unchanged.

Participants residing in the neighboring provinces of Bergamo and Brescia showed a higher infection rate than participants from the Milan province. These results reflect data from seroprevalence studies in Lombardy ${ }^{17,20}$. In line with other studies ${ }^{21,22}$, compared to married or cohabiting subjects, divorced and separated ones more frequently reported SARS-CoV-2 infection. However, the reasons for this finding are unclear.

The absence of chronic diseases was associated with a higher frequency of infection. This can be explained by a less cautious attitude of this sub-group compared to individuals with at least one chronic condition who were probably aware of the increased risks they were facing. In fact, the presence of specific chronic diseases leads to a depression of the immune system ${ }^{23}$ and an increased risk of COVID19 resulting in adverse outcomes ${ }^{5}$. This has been shown particularly among patients with diabetes. By contrast, patients reporting having been diagnosed with some forms of chronic migraine before the COVID-19 pandemic showed a higher infection rate.

Both COVID-19 and Post COVID-19 Syndrome can occur with migraine ${ }^{24,25}$, however our results seems to suggest that this condition could also result in an increased risk of infection. Further studies are needed to clarify this relationship.

A higher frequency of infection was observed in subjects reporting a worsening of depressive symptoms during the pandemic. Evidence from the literature shows that people who suffered from COVID-19 frequently experienced a deterioration of their mental health ${ }^{25-28}$. Furthermore, people who reported being infected most likely had other cases in their family ${ }^{29}$ and may have lost relatives as a result of COVID-19. The likelihood of reporting signs and symptoms referrable to COVID-19 may be related to the presence of depression and/or anxiety: studies comparing the prevalence of anxiety and depression among subjects who tested positive and subjects who tested negative to SARS-CoV-2 infection found different results ${ }^{30,31}$, so further research is needed to understand the nature of this relationship.

Our findings should be interpreted in light of some limitations. The first one to mention is that data on diagnostic tests and COVID-19-like signs and symptoms were self-reported, this exposes to potential recall bias. Furthermore, it must be considered that testing capacity in Italy changed during 2020 and that some participants may have passed through an asymptomatic SARS-CoV-2 infection without ever being tested. Moreover, as this is a cross-sectional study, we could not establish any causal relationship, although all efforts have been considered to give a longitudinal context to the analyses. However, to the 
best of our knowledge, this is the first study that investigates the role of possible determinants of SARSCoV-2 infection in older people. We also believe that this study provides useful elements to obtain a more reliable estimate of the magnitude of the cumulative incidence of COVID-19 in this population than data on nasopharyngeal swabs alone. Furthermore, we used a large representative sample of the population aged 65 or more from the Lombardy Region, and data were collected through a telephone-based interview, which can be considered the most suitable solution for the older population in a pandemic context. In fact, during the pandemic, online surveys proved to be an effective tool for overcoming social distancing measures ${ }^{32}$, but they carry the risk of selection bias since they exclude those who, like many seniors, have poor digital literacy and internet access ${ }^{33}$.

In conclusion, our results suggest that the oldest old subjects and those having chronic conditions limited their exposure to SARS-CoV-2 infection. Although the COVID-19 case fatality rate increases dramatically after the age of $70^{34}$, our study seems to indicate that those between the ages of 65 and 69 are more susceptible to SARS-CoV-2 infection and should be targeted by preventive strategies to better contain the spread of contagion.

\section{Methods}

A telephone-based cross-sectional survey was performed by Doxa, the Italian division of the Worldwide Independent Network/Gallup International Association, and coordinated by Mario Negri Institute and other Italian universities and research institutions ${ }^{35}$. The LOckdown and lifeSTyles in Lombardia (LOST in Lombardia) study has been conducted between November 17th and 30th 2020, on a representative sample of 4,400 older adults (aged 65 and over) from Lombardy region (Northern Italy) ${ }^{36}$.

Participants were randomly selected within a list of approximately 30,000 families, representative, by province and size of municipality, of the families from Lombardy region. A quota method was applied to ensure the representativeness of the sample in terms of sex, age, and province of residence.

The study protocol was approved by the ethics committee (EC) of the coordinating group (EC of Fondazione IRCCS Istituto Neurologico Carlo Besta, File number 76, October 2020). All participants provided their informed consent to participate to the study. All methods were performed in accordance with the relevant guidelines and regulations of Scientific Reports and of Nature Research journals.

Participants provided information on socio-demographic characteristics (such as sex, age, level of education, province of residence), marital status, and the presence of selected chronic conditions before the pandemic onset (reference time: November 2019).

Anxiety and depressive symptoms were assessed using the two-item Generalized anxiety disorder scale $(\mathrm{GAD}-2)^{37}$ and the two-item patient heath questionnaire (PHQ-2) ${ }^{38}$, respectively. In order to evaluate any potential changes in psychological wellbeing, the presence of anxiety and depressive symptoms was asked twice, referring to the time of the interview (November 2020) and one year before (November 2019). 
Respondents reported if they had been infected by SARS-CoV-2 and the type of diagnosis they received: a positive rhino pharyngeal swab test, a positive serological test or if they thought they had experienced signs or symptoms attributable to COVID-19 without a confirmed diagnosis. We defined this measure as history of SARS-CoV-2 infection.

\section{Statistical Analysis}

To investigate the determinants of SARS-CoV-2 infection, we estimated odds ratios (OR) and their corresponding $95 \%$ confidence intervals $(\mathrm{Cl})$ thorough unconditional multiple logistic models. As a sensitivity analysis, we re-run all the models excluding subjects reporting only signs or symptoms but who had not received a diagnosis. We defined a worsening in anxiety and depressive symptoms as any increase in the corresponding scales. All the models were adjusted for sex, age, level of education, province and number of comorbidities. A statistical weight was applied to all the analyses to guarantee the representativeness of the sample in terms of sex, age and province of residence.

All statistical analyses were performed using SAS 9.4 (Cary, North Carolina, USA).

\section{Declarations}

\section{LOST in Lombardia Project Investigators (alphabetical order)}

Andrea Amerio ${ }^{1}$, Mario Amore ${ }^{1}$, Marialaura Bonaccio ${ }^{2},{ }^{3}$, Cristina Bosetti ${ }^{4}$, Luca Cavalieri d'Oro ${ }^{5}$, Roberta Ciampichini ${ }^{6}$, Roberto De Sena ${ }^{7}$, Silvano Gallus ${ }^{4}$, Simone Ghislandi ${ }^{7}$, Francesco Gianfagna ${ }^{2,8}$, Licia lacoviello ${ }^{2}{ }^{3}$, Carlotta Micaela Jarach ${ }^{4}$, Alessandra Lugo ${ }^{4}$, Giansanto Mosconi ${ }^{9}$, Anna Odone ${ }^{9}$, Magda Rognoni ${ }^{5}$, Claudia Santucci ${ }^{4}$, Marco Scala ${ }^{5}$, Gianluca Serafini ${ }^{1}$, Carlo Signorelli $^{9}$, Chiara Stival ${ }^{4}$, David Stuckler ${ }^{7}$, Giacomo Pietro Vigezzi ${ }^{9}$, Yuxi Wang ${ }^{7}$, Alberto Zucchi ${ }^{6}$

${ }^{1}$ Università di Genova, Genoa, Italy

${ }^{2}$ Università degli Studi dell'Insubria, Varese, Italy

${ }^{3}$ IRCCS Neuromed, Pozzilli, Italy

${ }^{4}$ Istituto di Ricerche Farmacologiche Mario Negri IRCCS, Milan, Italy

${ }^{5}$ Agenzia per la Tutela della Salute della Brianza, Monza, Italy

${ }^{6}$ Agenzia per la Tutela della Salute di Bergamo, Bergamo, Italy

${ }^{7}$ Università Bocconi, Milan, Italy

${ }^{8}$ Mediterranea Cardiocentro, Napoli, Italy 
${ }^{9}$ Università di Pavia, Pavia, Italy and Università Vita-Salute San Raffaele, Milan, Italy

\section{Author contributions statements}

GM, CS, SG, and AO conceptualized and designed the study. CS analysed the data under the supervision of SG. GM wrote the first draft of the manuscript in collaboration with CS, and SG. All other authors provided important contributions for the interpretation of findings. AO, and SG provided supervision and important intellectual support in various steps of the study. All authors have read and approved the last version of the manuscript.

\section{Data availability statement}

Data that support findings of this study and materials are available from the corresponding author, AO, upon reasonable request.

\section{Declaration of Sources of Funding}

This work was supported by the DG-Welfare of Lombardy Region (Call: Progetti di ricerca in ambito sanitario connessi all'emergenza COVID-19; DGR n. XI/3017); and AXA (AXA Research Fund - Call for Proposals COVID-19). The work of CS, AL and SG is partially supported by the Italian League Against Cancer (LILT, Milan).

The funders played no role in the study design, data collection, analysis, decision to publish or preparation of the manuscript.

\section{Declaration of Conflicts of Interest}

None.

\section{References}

1. Odone, A., Delmonte, D., Scognamiglio, T. \& Signorelli, C. COVID-19 deaths in Lombardy, Italy: data in context. The Lancet Public Health (2020) doi:10.1016/S2468-2667(20)30099-2.

2. Remuzzi, A. \& Remuzzi, G. COVID-19 and Italy: what next? The Lancet vol. 395 (2020).

3. Perico, L. et al. COVID-19 and lombardy: TESTing the impact of the first wave of the pandemic. EBioMedicine 61, (2020).

4. Istituto Superiore di Sanità. Epidemia COVID-19: Aggiornamento nazionale 29 dicembre 2020. https://www.epicentro.iss.it/coronavirus/bollettino/Bollettino-sorveglianza-integrata-COVID-19_29dicembre-2020.pdf (2020).

5. Zheng, Z. et al. Risk factors of critical \& mortal COVID-19 cases: A systematic literature review and meta-analysis. Journal of Infection vol. 81 (2020). 
6. Doraiswamy, S., Mamtani, R., Ameduri, M., Abraham, A. \& Cheema, S. Respiratory epidemics and older people. Age Ageing 49, 896-900 (2020).

7. Li, J. et al. Epidemiology of COVID-19: A systematic review and meta-analysis of clinical characteristics, risk factors, and outcomes. J. Med. Virol. 93, 1449-1458 (2021).

8. Zambianchi, M., Rönnlund, M. \& Carelli, M. G. Attitudes Towards and Use of Information and Communication Technologies (ICTs) Among Older Adults in Italy and Sweden: the Influence of Cultural Context, Socio-Demographic Factors, and Time Perspective. J. Cross. Cult. Gerontol. 34, 291-306 (2019).

9. Istituto Superiore di Sanità. Epidemia COVID-19: Aggiornamento nazionale 25 novembre 2020. https://www.epicentro.iss.it/coronavirus/bollettino/Bollettino-sorveglianza-integrata-COVID-19_25novembre-2020.pdf (2020).

10. Bassi, F., Arbia, G. \& Falorsi, P. D. Observed and estimated prevalence of Covid-19 in Italy: How to estimate the total cases from medical swabs data. Sci. Total Environ. 764, (2021).

11. Istituto Nazionale di Statistica; Italian Ministry of Health. Primi risultati dell'indagine di sieroprevalenza sul SARS-CoV-2. https://www.istat.it/it/archivio/246156 (2020).

12. Fu, L. et al. Clinical characteristics of coronavirus disease 2019 (COVID-19) in China: A systematic review and meta-analysis. J. Infect. 80, 656-665 (2020).

13. Horsley, J. R., Sterling, I. J. N., Waters, W. E. \& Howell, J. B. L. Respiratory Symptoms among Elderly People in the New Forest Area as assessed by Postal Questionnaire. Age Ageing 20, 325-331 (1991).

14. Dow, L. et al. Respiratory symptoms in older people and use of domestic gas appliances. Thorax $\mathbf{5 4}$, 1104-1106 (1999).

15. Pijls, B. G. et al. Demographic risk factors for COVID-19 infection, severity, ICU admission and death: A meta-analysis of 59 studies. BMJ Open 11, (2021).

16. Rostami, A. et al. Update on SARS-CoV-2 seroprevalence: regional and worldwide. Clin. Microbiol. Infect. (2021) doi:10.1016/J.CMI.2021.09.019.

17. Gallus, S. et al. SARS-CoV-2 Infection among the Dental Staff from Lombardy Region, Italy. Int. J. Environ. Res. Public Health 18, (2021).

18. IZA - Institute of Labor Economics. Loneliness and Social Isolation: An Unequally Shared Burden in Europe | IZA - Institute of Labor Economics. https://www.iza.org/publications/dp/14245/lonelinessand-social-isolation-an-unequally-shared-burden-in-europe (2021).

19. Fadel, T., Travis, J., Harris, S. \& Webb, G. The roles of experiences and risk perception in the practice of preventative behaviors of COVID-19. https://doi.org/10.1080/20477724.2021.1957595 (2021) doi:10.1080/20477724.2021.1957595.

20. Signorelli, C. et al. Epidemiological assessment of the first COVID-19 epidemic wave in Lombardy. A systematic review. Acta Biomed. 92, (2021).

21. Pritsch, M. et al. Prevalence and Risk Factors of Infection in the Representative COVID-19 Cohort Munich. Int. J. Environ. Res. Public Health 18, (2021). 
22. Napolitano, F. et al. Seroprevalence of SARS-CoV-2 Antibodies in Adults and Healthcare Workers in Southern Italy. Int. J. Environ. Res. Public Health 18, (2021).

23. Furman, D. et al. Chronic inflammation in the etiology of disease across the life span. Nat. Med. 2019 2512 25, 1822-1832 (2019).

24. Misra, S. et al. Frequency of Neurologic Manifestations in COVID-19: A Systematic Review and Metaanalysis. Neurology 10.1212/WNL.0000000000012930 (2021) doi:10.1212/WNL.0000000000012930.

25. Malik, P. et al. Post-acute COVID-19 syndrome (PCS) and health-related quality of life (HRQoL)-A systematic review and meta-analysis. J. Med. Virol. 94, 253-262 (2022).

26. Renaud-Charest, O. et al. Onset and frequency of depression in post-COVID-19 syndrome: A systematic review. J. Psychiatr. Res. 144, 129-137 (2021).

27. Amerio, A. et al. Covid-19 pandemic impact on mental health of vulnerable populations. Acta Bio Medica Atenei Parm. 91, 95 (2020).

28. Amerio, A. et al. COVID-19 lockdown impact on mental health in a large representative sample of Italian adults. J. Affect. Disord. 292, 398-404 (2021).

29. Ng, O. T. et al. SARS-CoV-2 seroprevalence and transmission risk factors among high-risk close contacts: a retrospective cohort study. Lancet. Infect. Dis. 21, 333-343 (2021).

30. Zhang, J. et al. The differential psychological distress of populations affected by the COVID-19 pandemic. Brain. Behav. Immun. 87, 49 (2020).

31. Paz, C. et al. Anxiety and depression in patients with confirmed and suspected COVID-19 in Ecuador. Psychiatry Clin. Neurosci. 74, 554-555 (2020).

32. Hlatshwako, T. G. et al. Online health survey research during COVID-19. Lancet Digit. Heal. 3, e76e77 (2021).

33. Zambianchi, M., Rönnlund, M. \& Carelli, M. G. Attitudes Towards and Use of Information and Communication Technologies (ICTs) Among Older Adults in Italy and Sweden: the Influence of Cultural Context, Socio-Demographic Factors, and Time Perspective. J. Cross. Cult. Gerontol. 34, (2019).

34. Onder, G., Rezza, G. \& Brusaferro, S. Case-Fatality Rate and Characteristics of Patients Dying in Relation to COVID-19 in Italy. JAMA 323, 1775-1776 (2020).

35. Odone, A. et al. COVID-19 lockdown impact on lifestyle habits of Italian adults. Acta Biomed. Atenei Parm. 91, 87-89 (2020).

36. Istituto Nazionale di Statistica. Popolazione residente al $1^{\circ}$ gennaio: Lombardia. http://dati.istat.it/Index.aspx?Queryld=18548\# (2020).

37. Spitzer, R. L., Kroenke, K. \& Williams, J. B. Validation and utility of a self-report version of PRIME-MD: the PHQ primary care study. Primary Care Evaluation of Mental Disorders. Patient Health Questionnaire. JAMA 282, (1999). 
38. Kroenke, K., Spitzer, R. L. \& Williams, J. B. W. The patient health questionnaire-2: Validity of a twoitem depression screener. Med. Care 41, (2003).

\section{Figures}

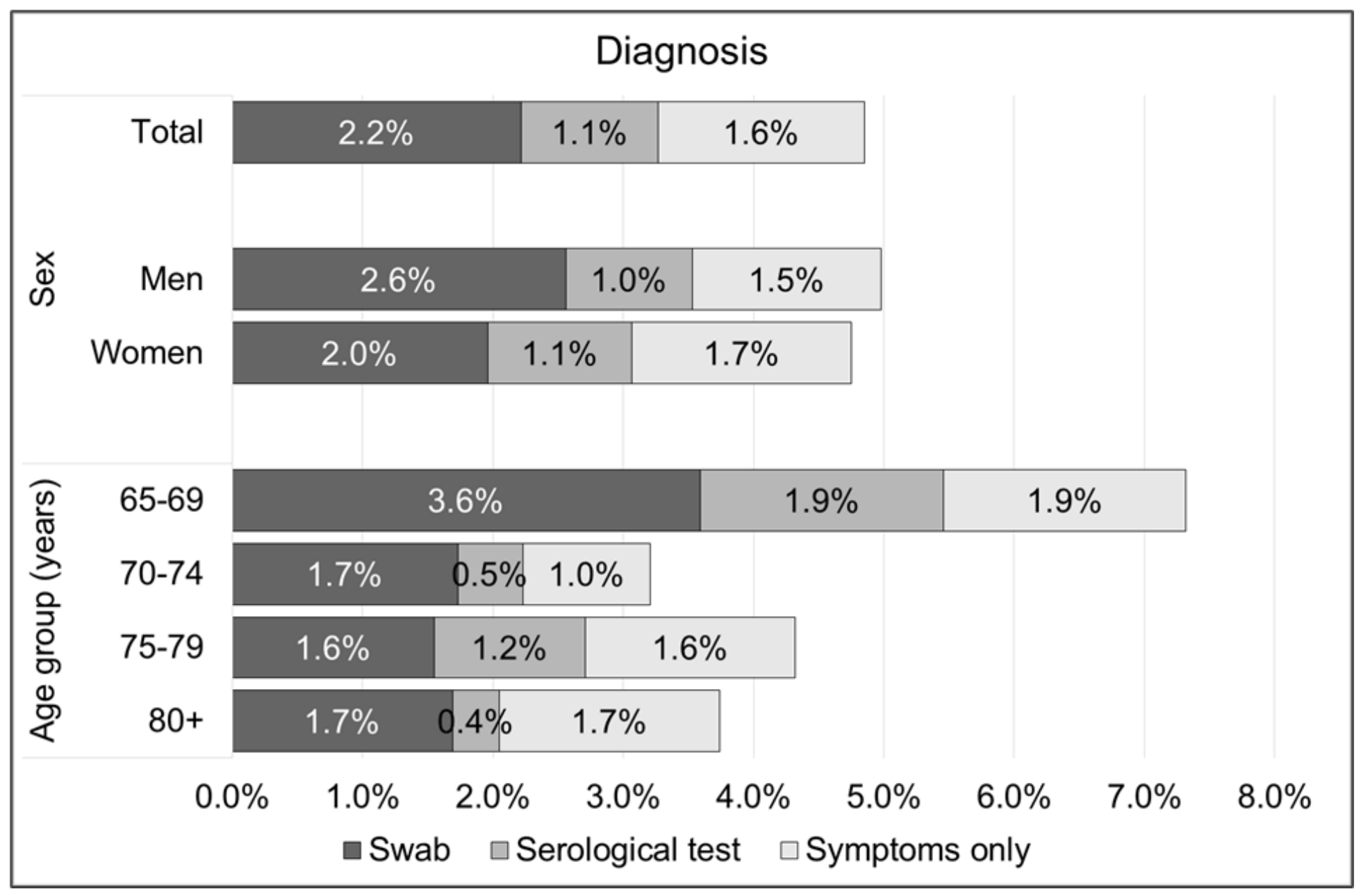

\section{Figure 1}

Distribution of 4400 Italian subjects aged 65 years or more according to COVID-19 infection and type of diagnosis, overall and by sex and age group. Lombardy region, Italy 2020.

\section{Supplementary Files}

This is a list of supplementary files associated with this preprint. Click to download.

- Supplementarytable1.docx 